\title{
Surveillance Data Analysis of Typhoid Fever in Jimma Zone, Oromia Region, Ethiopia (2015 - 2019)
}

Getaneh Atikilt Yemata ( $\square$ gechat9@gmail.com )

University/College Library https://orcid.org/0000-0002-0879-3303

\section{Chalachew Yenew}

Debre Tabor University

Melkalem Mamuye

Debre Tabor University

Mulu Tiruneh

Debre Tabor University

Tiagabinesh Assfaw

Debre Tabor University

Sileshi Mulatu

Bahir Dar University

Ermias Sisay

Debre Tabor University

Research

Keywords: Surveillance, Typhoid Fever, Jimma Zone

Posted Date: August 17th, 2021

DOI: https://doi.org/10.21203/rs.3.rs-720256/v1

License: (c) (1) This work is licensed under a Creative Commons Attribution 4.0 International License.

Read Full License 


\section{Abstract}

Introduction

Typhoid fever is a major cause of morbidity and mortality around the globe and it is a serious illness in developing countries. Typhoid fever is prevalent in Ethiopia and the burden differs with diverse demography, environment, and climate. Efficient evidence concerning the trend of typhoid fever is important for targeted intervention in the catchment area. So, ongoing analysis of surveillance data is vital but there is no data regarding typhoid fever trend and distribution in the study area. The study aimed to determine the proportion of typhoid fever cases by person, district, and time ,and to assess the mortality rate of typhoid fever.

Method

Descriptive cross-sectional study design was conducted among monthly reported typhoid fever surveillance data of 5 years (2015-2019) in Jimma Zone. The data were retrieved, analyzed, and interpreted from May 20/2019 to June $7 / 2019$. The study population is estimated to be 3425206 . SPSS version 21 was used to enter and analyze the data.

Result

A total of 36641 typhoid fever cases were reported. The total prevalence rate during the five years was 24 cases per10,000 residents. The disease mostly occurred in March with these $7200(20 \%)$ reported during the period. The majority of the investigated cases were identified in Qarsa woreda 4476(12.2\%), Followed by Gomma $4075(11.1 \%)$ and Mana 3267 (8.9\%) wereda. $159(0.4 \%)$ of the reported cases were admitted and the majority 60 (37.7\%) were from Gumay wereda but there was no death from all wereda in the five years.

Conclusion and Recommendation

Typhoid fever was a major public health problem in the Jimma zone for the last 5 years. The highest incidence of typhoid fever cases was reported at the end of the dry season particularly in March and the distribution of cases was positively skewed to Qarsa, Gomma, and Mana wereda among the surveillance areas. Zonal health departments should strengthen the interventions focused on the wereda that had a high burden of typhoid fever at the end of the dry season.

\section{Introduction}

Typhoid Fever(TF) is a major cause of morbidity and mortality around the globe and it is a serious illness in developing countries. Reports that estimate the global burden of typhoid fever indicate that the disease is still a public health issue. Annually in the globe, there are around 17 million, typhoid fever cases and more than a half-million deaths. In developing countries, around 12.5 million persons are affected by typhoid fever each year $(1,2)$. 
Typhoid fever is one of the public health significant diseases in low-income countries like Ethiopia in which the safety of drinking water supply, hygienic condition, and quality of life are poor and below standard. If left untreated typhoid fever is a lethal disease where $10-30 \%$ of cases will end in death. While with intervention the case fatality rate will be dropped to $1-4 \%(3)$.

In Ethiopia, similar to other low-income countries, there was a challenge to evaluate and determine the burden of typhoid fever cases since inadequate coverage of studies and dearth of coordinated surveillance systems in the country. Besides, a low report rate of positive cases and the occurrence of other infectious diseases that gets high importance in the surveillance system may make the problem of typhoid fever appear small by comparison and lacks concern(1).

According to the Ethiopia Federal Minister Of Health $(\mathrm{FMOH})$ report, two percent of males and three percent of females died because of typhoid fever (4). Among the symptomatic cases of typhoid fever, around $10-40 \%$ will have a risk to be complicated and died if left untreated. Even from typhoid fever patients that had got treatment two to five percent of them will have a risk of death. In Ethiopia, typhoid fever is an endemic, and epidemic-prone disease especially in the area of highlands in which around 10,000 cases are reported each year. People without a home that lives in swarming and poor hygienic conditions are primarily affected by the disease(5).

Thus far FMOH has established different intervention strategies for the community to improve awareness and risk perception of infectious disease including typhoid fever through health extension workers. However, the burden of TF has not reduced to an acceptable rate(4).

Bearing in mind, in Ethiopia typhoid fever is an endemic disease, and the burden and consequence of the disease vary with different ecology, climate, and population groups. So Communicate the latest new information concerning the distribution of typhoid fever in the catchment area have valuable significance for early and prioritized interventions (6).

Typhoid fever is most common among individuals who work in food handling and preparation activities, overcrowding slums, and low economic status peoples because of poor hygiene and waste disposal system. Unless protective and control measures are taken appropriately, the outbreak of typhoid fever could occur (7).

Continuing investigation of surveillance data is significant for noticing disease outbreaks, judging disease tendencies, and evaluating the efficacy of disease prevention and control programs and strategies. In addition, the surveillance data analysis provides indicative information about the appropriate health resource allocation (8). Despite the presence of collected data in the zonal health departments, it is not analyzed by considering epidemiology of disease distribution in which it helps to know the at-risk population, trends over time, and the geographical distribution of the diseases which in turn helps for appropriate intervention (9). Therefore, this study aimed to analyze 5 years of reported typhoid fever surveillance data of the Jimma zone. 


\section{Methods}

Study Area

The Surveillance data analysis was employed in Jimma Zone which is one of the 20 rural zones in the Oromia region located in the South West of Ethiopia with a distance of $352 \mathrm{KM}$ from Addis Ababa. The total area of the zone is $199316.18 \mathrm{~km}^{2}$, accounting for about $17.2 \%$ of the total area of the region. Climatically the zone is classified into three agro-ecological zones, namely, highlands (14.93\%), midlands (21.54\%), and lowlands (63.53\%). The annual rainfall ranges from $900 \mathrm{ml}$ to $1400 \mathrm{ml}$. The annual mean temperature of the zone ranges from $10^{\circ} \mathrm{C}$ to $25^{\circ} \mathrm{C}$ with a mean temperature of $17.5^{\circ} \mathrm{C}$. Fifty-three percent of the zone is exposed to malaria. Administratively, the zone is divided into 21 administrative woredas (19 rural woreda and 2 towns) and 566 Kebeles.

According to the Ethiopian 2011, Population and Housing Census projection, the total population of the zone in July 2019 is estimated to be 3425206 with 1678351 males and 1746855 females. The average household size in the zone is 4.8 , with a population density of 32.10 , which greatly varies among zones and woredas. Likewise, Jimma Zone has 3 general hospitals, 5 primary hospitals, 122 health centers, 566 health posts, 10 private medium clinics, 101 lower clinics, 31 drug stores, and 41 rural drug vendors.

\section{Study Period}

Monthly reported of 5 years (2015-2019) typhoid fever surveillance data of Jimma Zone were retrieved, analyzed, and interpreted from May 20/2019 to June 3/ 2019.

\section{Study Design}

A descriptive cross-sectional study design was used to analyze retrospectively 5 years (2015-2019) data on the distribution of typhoid fever cases from monthly reportable typhoid fever surveillance data of the Jimma Zone health office.

\section{Population}

The source populations were all people at risk of typhoid fever in the Jimma Zone, Oromia region. All population of Jimma Zone that visits the health institution under surveillance in the zone and diagnosed with typhoid fever during 2015-2019 was a population of the study.

Inclusion criteria

Health center and Hospital that is in surveillance system

Study Variables

Distribution of typhoid fever in time, person, and place 


\section{Case definitions of typhoid fever}

Suspected: A person presented with gradual onset of remittent fever or fever rising in a stepladder fashion in the first week and manifested with arthralgia, headache, anorexia, abdominal pain, and constipation. Confirmed: A suspected case with Widal test, "O" titer of 1/160 and more, is very suggestive, A suspected case with positive blood culture at the 1 st week or positive stool culture at 3rd, 4th, and 5thweek illness are very definitive (2).

Data collection method

The data were collected through a document review of available sources by two field epidemiology professionals. The consecutive Five years data of Typhoid fever reports were collected from the zone health office (HMIS and PHEM department).

Data processing and analysis

Data were entered in Epi data version 3.1 and exported to SPSS version 21 for data cleaning and analysis. Baseline characteristics of study subjects were described using frequency and percentages. Distribution of typhoid fever in time (months and years), geography (Wereda), and persons (age and sex) were computed using descriptive statistics. Tables and graphs were used to describe the results. Finally, results were reported as frequency and percentages.

\section{Result}

\section{Number of facilities during 2015-2019}

In the Jimma zone, there was an increment in health service coverage by the end of 2018 (90\%) compared to 2015(15\%). Furthermore, there were 476 Health posts (HPs), 100 Health Centers (HCs), and 1 Hospital in 2015 when the zone was established as new, and now at the end of 2018 there were 531 HPs, 119 HCs, and 5 District Hospitals (Figure,1).

\section{Report Completeness}

Report completeness in the Jimma zone increased from 2015 to 2019 by $3 \%$. The completeness of reporting rate of the zone (from all types of health facilities) in 2015 and 2019 was $95 \%$ and $98 \%$ respectively (Table 1$)$.

Table 1: Zonal Surveillance report (from all health facilities) completeness by year in Jimma Zone, Oromia from 2015 to 2019 


\begin{tabular}{llll} 
Years & Expected & Report & Completeness \% \\
\hline 2015 & 577 & 521 & 95 \\
\hline 2016 & 642 & 581 & 95 \\
\hline 2017 & 643 & 542 & 97 \\
\hline 2018 & 644 & 545 & 98 \\
\hline 2019 & 653 & 625 & 98
\end{tabular}

Zonal disease trends

Distribution of Typhoid Fever by Time

In the last 5 years (2015 to 2019), Zonal Public Health Emergency Management Unit (PHEM) received 36641 reports concerning cases of Typhoid Fever among persons in the Jimma zone. The mean was 6092 cases per year, ranges between 5874 and 9899. The highest number of cases (9899) and percent of cases (27\%) were recorded in 2019 (Table 2).

Table 2 : Trends of Typhoid Fever by Year in Jimma Zone, Oromia Region, South West Ethiopia from 2015 to 2019

\begin{tabular}{lllllll} 
Years & Population at risk & $\begin{array}{l}\text { Number of } \\
\text { Cases }\end{array}$ & $\begin{array}{l}\text { Number of } \\
\text { death }\end{array}$ & Percent of case & $\begin{array}{l}\text { Prevalence } \\
\text { (Case/10000) }\end{array}$ & CFR \\
\hline 2015 & $2,863,762$ & 6182 & 0 & $17 \%$ & 22 & 0 \\
\hline 2016 & $2,976,050$ & 5874 & 0 & $16 \%$ & 20 & 0 \\
\hline 2017 & $3,088,338$ & 6265 & 0 & $17 \%$ & 21 & 0 \\
\hline 2018 & $3,200,626$ & 8421 & 0 & $23 \%$ & 27 & 0 \\
\hline 2019 & $3,312,914$ & 9899 & 0 & $27 \%$ & 30 & 0 \\
\hline Total & $3,088,338$ & 36641 & & & 24 &
\end{tabular}

During the five consequent years, the prevalence rate of typhoid fever increased from 22 per 10,000 cases in 2015 to 30 cases per 10,000 in 2019 . The uppermost prevalence rate was reported during 2019. The total prevalence rate during the five years among the target population (mid-term population) was 24 cases per 10,000 residents. According to the surveillance data reported, there was no death due to typhoid fever during the five consecutive years (Figure 2).

\section{Distribution of Morbidity by Month vs. Years}

From a total of typhoid fever cases $(36,641)$ during the five years, there was a report in all months. The highest number of cases (910) were reported during March 2019. The highest number of cases (peak) per 
month in each year was recorded as in 2015 it was in March and September, in 2016 during March and December, in 2017 during March, June, and September, in 2018 during November, June, and August and in 2019 during October, March, and June. There was a constantly increasing number of cases from November to March in 2015, 2017, and 2019. Furthermore, there was a small peak in all years during a march, except in 2018. However, there was no constant peak time throughout the 5 years. Overall, the incidence was not uniform, but it was on an increasing mode in some months, and September, November, October, March, and June were the months in which the highest number of cases were recorded (Figure 3 ) .

\section{Distribution of typhoid Fever in Place or Wereda}

When we see the distribution of typhoid fever cases by woredas during the five years, which means from 2015 to the end of 2019, the Zonal PHEM unit received surveillance reports from 21 woredas. From the total report of 36641 typhoid fever cases, the majority of the report was from Qarsa woreda 4476(12.2\%), Followed by Gomma 4075(11.1\%) and Mana 3267 (8.9\%). Moreover, all Hospitals reported the lowest cases, which is $0 \%$ to $1 \%$ within the last five years (figure 4 ).

Distribution of Morbidity by Woreda / place

Of 159 admitted cases the majority of them were reported from Gumay woreda 60 (37.7\%) and around ten woredas have no admission of typhoid fever. However, no mortality occurs at all (Figure 5).

\section{Distribution of Typhoid Fever by person}

A total of 36641 Typhoid fever cases were registered from 2015 to 2019. Among this, 18972 (51.8\%) were females and 17669 (48.2\%) were males (Figure 6). The highest average annual incidence of typhoid fever was observed among study subjects with the age category of above 19 years (25307[69\%]) followed by the age category of above 9 years to 19 years $10333(28.2 \%)$. No death occurred in all age groups (Figure 6 and 7).

\section{Discussion}

This study examined the completeness of typhoid fever incidence indicator reporting in the PHEM system in the Jimma zone between 2015and 2019 and it was greater than the standards of the national target $(80 \%)$. The study concluded that it has good quality to offer an assessment of typhoid fever distribution.

The total prevalence of typhoid fever cases in the five years was 24 per 10,000 persons. When we see the prevalence rate in each year among the total target population,22 cases in 2015,20 cases in 2016,21 cases in 2017,27 cases in 2018, and 30 cases in 2019 per 10,000 inhabitants were investigated. The result suggested that the burden of typhoid fever cases was increased from 2015 to 2019 with a fluctuating trend, even though different interventions have been implemented in the catchment area. The trend is congruent with a study in Lalo Asabi district West Wollega, Ethiopia which was increasing in instability mode(10). It could be due to the expansion of hospitals and health centers and health service 
coverage through consecutive years in the Jimma Zone, which can investigate an increased number of typhoid fever cases.

The result of our study was lower as compared to the national health and health-related indicator report, with 34 and 39 cases per 10000 inhabitants in the Oromia region and the national level(11). While the finding of our study is much higher as compared to a study in Nigeria (6.3\%)(12). The discrepancy could be justified due to either the attempt of the health care providers including health extension workers in the area or the validity of a diagnostic test. In Ethiopia, mostly performed diagnosis for typhoid fever is the Widal test, which measures antibodies against ' $\mathrm{O}$ ' and ' $\mathrm{H}$ ' antigens of salmonella Typhi. The test lacks specificity and increases false-positive results that lead to over-diagnosis especially in endemic areas.

This result suggested that the magnitude of typhoid fever rises at the end of the dry season (March). But typhoid fever cases seasonal occurrence is not evidenced with other studies. In our study area context end of the dry season is the time in which, the rural water supply is lowest and people congregate at the source of water. This may be rain start this month and people living in the rural area utilize runoff water or pond water for drinking and cooking food without water treatment. At this time the rain helps spread already contaminated water supplies (5). Therefore, the opportunity of contamination of water with pathogenic organisms is high with the aid of poor sanitation practice,(1).

The result of this study concerning the distribution of typhoid fever cases in geographical areas or woredas in Jimma Zone concluded that the majority of the investigated cases were identified in Kersa (12\%), Gomma (11\%), Mana (9\%), Sigma, Setema, and Deedo wereda. Typhoid fever is more common in areas with poor sanitation practices (3), so unsafe and inadequate water for drinking and handwashing especially related to the toilet could be responsible for the increment of cases in the identified sites. This could generate the chance of transmission of the pathogen from one person to another person.

In our study, the admission rate in the five consecutive years was $0.4 \%$ and most of the admitted cases were reported in Gumay wereda (37.7\%) even though the prevalence of typhoid fever cases in this woreda was lower (3.1\%) as compared to other woredas. The case fatality rate was zero within 5 years. The morbidity and mortality of typhoid fever in the catchment area were minimal. This could be explained through the increasing awareness and health-seeking behavior, early detection of cases, appropriate diagnosis, and treatment that contribute to the minimization of inpatient cases and the absence of death.

Concerning the distribution of typhoid fever in sex the result of this study showed that females accounted for $51.8 \%$ of cases, which is congruent as we compare to a gender-based analysis of morbidity report of FMOH EFY, $52 \%$ of females encountered the cases(13). Most (69.1\%) of study subjects that have been affected with typhoid fever were in the age category of above 19 years followed by 9-19 years, and below 9 years respectively. This indicated that adults were more vulnerable than youths as compared to children. Our result is congruent with a study employed in Arba Minch health centers, Southern Ethiopia which showed that $52.3 \%, 47.4 \%$ of cases were female and male respectively. Besides, regarding the age category, 5-15 and 16-30 age of study subjects was highly affected as compared to study subjects of age 
less than five years (14). This implied that the proportion of typhoid fever in adults was higher as compared to pediatrics age groups.

\section{Limitation}

Most of the private clinics were not included in this surveillance data analysis because they were not in the government reporting system line. Therefore, the number of cases could be greater.

\section{Conclusion}

Typhoid fever was an important public health issue in the Jimma zone for the last 5 years and the increment of typhoid fever case incidence through the years was reported. In general, the majority of Typhoid fever cases were treated at the outpatient department and few cases were treated at the inpatient department. The highest incidence of typhoid fever cases was reported at the end of the dry season particularly in March. The highest incidence of typhoid fever cases was reported in Qarsa wereda during 2019. Besides adults were more more vulnerable for typhoid fever

\section{RECOMMENDATION}

This study aims to show the magnitude and distribution of typhoid fever cases in the Jimma zone and offer evidence for the appropriate management and decision on the strategy for concerned bodies, particularly local health system administrators.

To avert the burden and consequences of the disease, health care providers should deliver intensive health promotion programmers like safe hygienic conditions particularly handwashing related to cooking, eating, and toilet. Zonal health departments should strengthen the interventions focused on the wereda in which had a high burden of typhoid fever and females at the end of the dry season. Private health facilities or clinics must be included in the surveillance reporting system.

\section{Abbreviations}

EFY: Especially for Youth

FMOH: Federal Ministry of Health

PHEM: Public Health Emergency Management

WHO: World health organization

\section{Declarations}

Ethical Consideration 
The study was approved by Jimma University, the college of medicine, and the public health ethical review board. Permission was obtained from the Jimma Zone health department before data collection. For confidentiality, any information related to personnel data was collected using codes, and analysis was done leaving the codes. The methods and procedures were conducted according to Jimma university regulations and guidelines.

\section{Consent for publication}

Not applicable

\section{Availability of data and supplementary materials}

The data sets in this study are accessible from the corresponding author on reasonable request

\section{Competing interests}

The authors declare no competing interests

\section{Funding}

No fund was obtained for this particular study

\section{Authors' contributions}

G.A. and C.Y. wrote the manuscript including tables and figures. S.M., M.M., M.T, T.A., and E.S. have contributed to methods. The final manuscript is read and approved by all authors

\section{Acknowledgments}

The authors would like to acknowledge Jimma University, College of medicine, and public health for approving the ethical review process. The authors are also wanted to express thankfulness to data collectors, Jimma zonal health office staff, supervisors, and study participants.

\section{References}

1. Andrew K, Nguri B. RISK FACTORS INFLUENCING TYPHOID FEVER OCCURRENCE AMONG. 2011;

2. Gebreyesus A, Negash L. International Journal of Infectious Diseases Diagnosis and Treatment of Typhoid Fever and Associated Prevailing Drug Resistance in Northern Ethiopia. Int J Infect Dis [Internet]. 2015;35:96-102. Available from: http://dx.doi.org/10.1016/j.ijid.2015.04.014

3. World Health Organization. Vaccine-Preventable Disease; Typhoid and another invasive salmonellosis. 
4. Yehualashet SS. Assessment of Knowledge and Risk Perception towards Typhoid Fever among Communities in Mendida Town. 2018;

5. Prevalence of Typhoid Fever in Oromia Region, West Arsi Zone from July 2007 to June 2012. 2013; (July 2007).

6. Habte L, Tadesse E, Ferede G, Amsalu A. Typhoid fever: clinical presentation and associated factors in febrile patients visiting Shashemene Referral Hospital, southern. BMC Res Notes [Internet]. 2018;1-6. Available from: https://doi.org/10.1186/s13104-018-3713-y

7. Feasey NA, Archer BN, Heyderman RS, Sooka A, Dennis B, Gordon MA, et al. Typhoid Fever and Invasive Nontyphoid Salmonellosis, Malawi and South Africa. 2010;16(9):1448-51.

8. Jima D, Wondabeku M, Alemu A, Teferra A, Awel N, Deressa W, et al. Analysis of malaria surveillance data in Ethiopia: what can be learned from the Integrated Disease Surveillance and Response System? BioMed Cent. 2012;1-14.

9. Zerfu B, Medhin G, Mamo G, Getahun G, Tschopp R, Id ML. Community-based prevalence of typhoid fever, typhus, brucellosis, and malaria among symptomatic individuals in Afar Region, Ethiopia. PLOS Neglected Trop Dis. 2018;80:1-16.

10. Eba K, Bekele D. Prevalence of Typhoid Fever and its Risk Factors in Lalo Assabi. 2019;10:1-8.

11. Ethiopia Federal Ministry Of Health. Health and health-related indicators, 2011/12. 2012;

12. Assessment of Knowledge and Risk Perception towards Typhoid Fever among Communities in Mendida Town, Ethiopia 2018 EC PAEDIATRICS Research Article. 2018;12:1141-57.

13. WHO, UNICEF, UNFPA WBG, and the UNPD. Indicators of Sustainable Development: Guidelines and Methodologies - Third edition Methodology sheets. 2007.

14. Lemi BW. Typhoid Fever in an Ethiopian Health Center. J Microbiol Infect Dis. 2019;9(November):150-4.

\section{Figures}




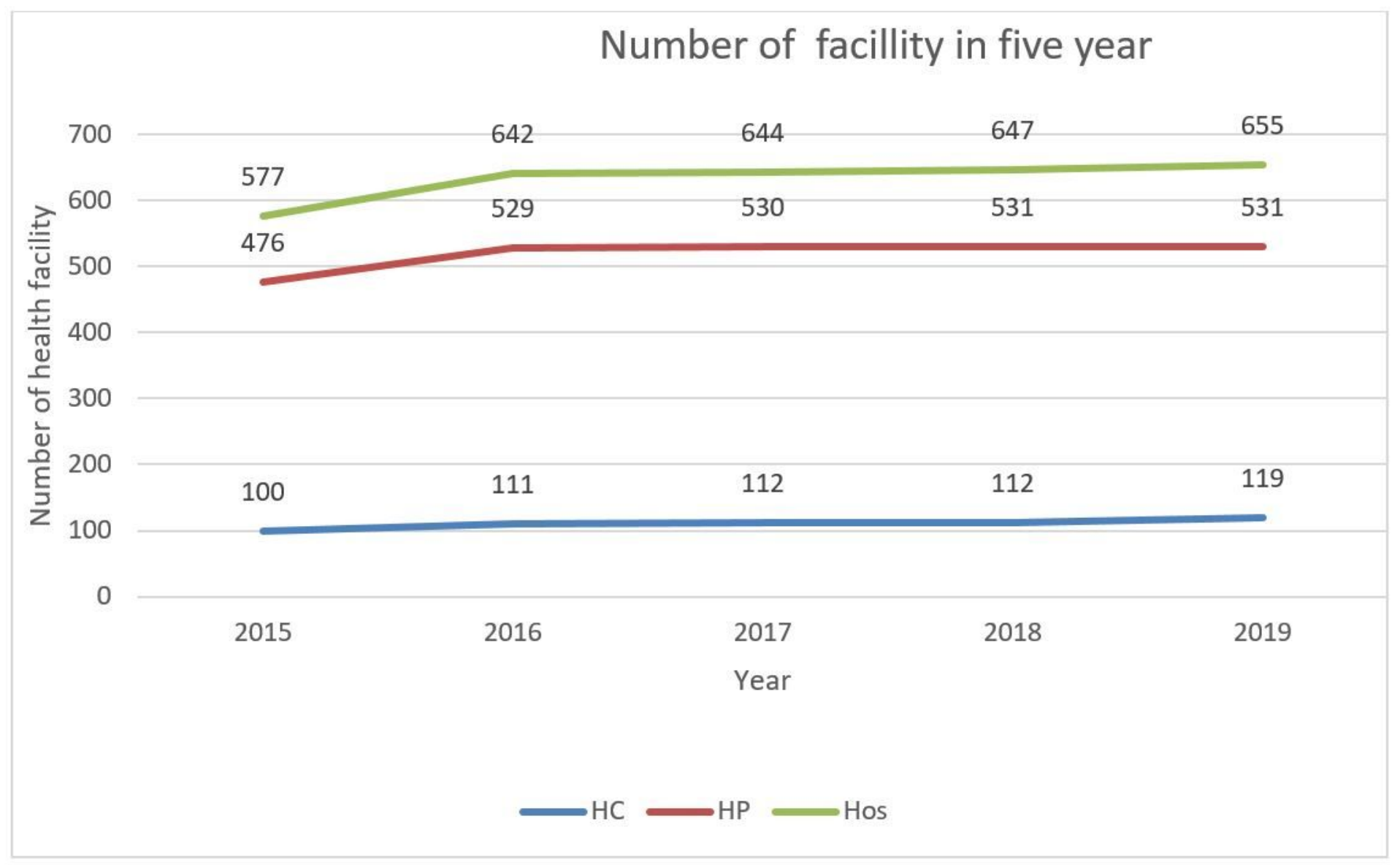

\section{Figure 1}

Number of Health Facilities expected to report by year in Oromia Region, Jimma zone from 2015 to 2019 


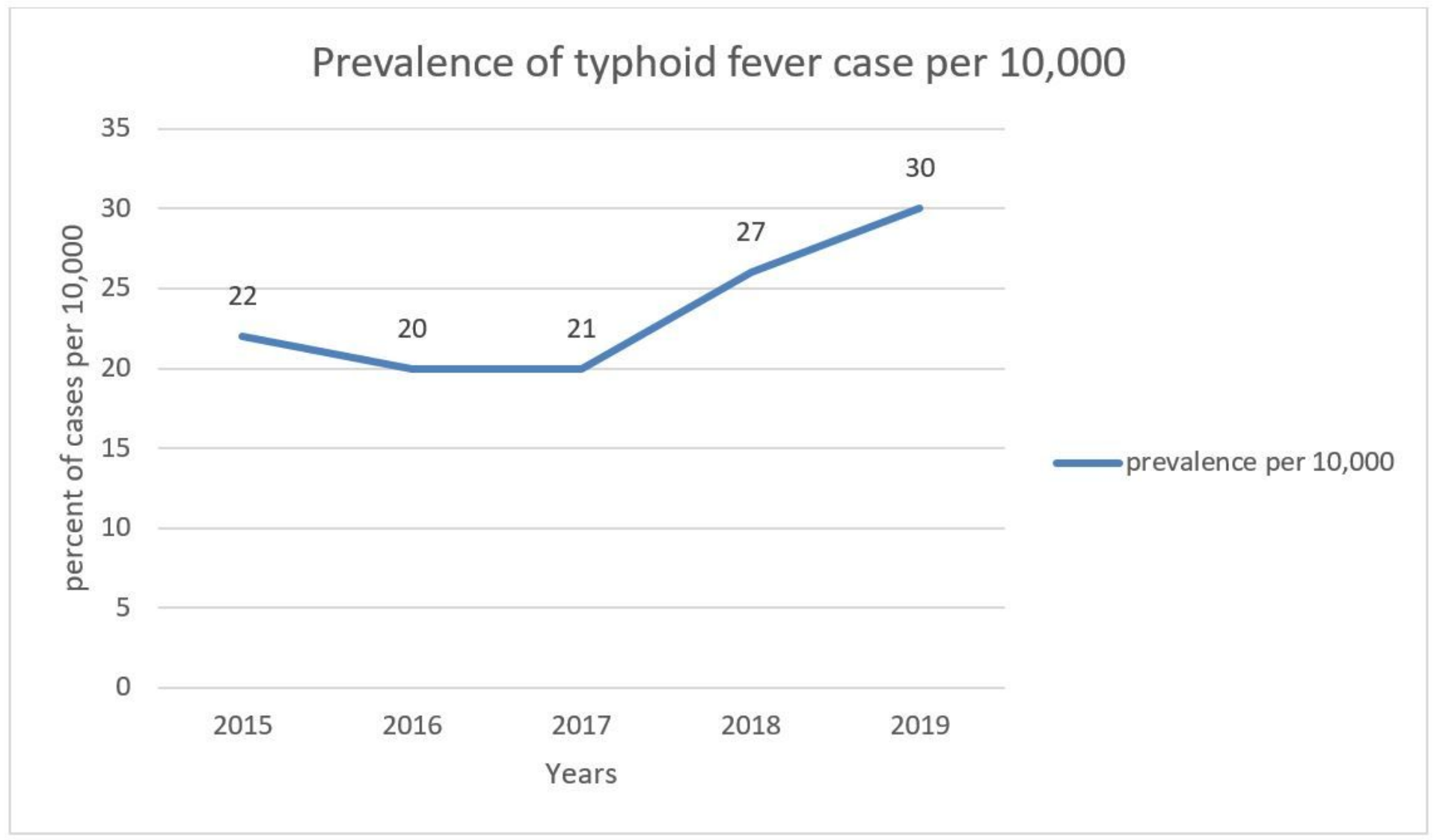

Figure 2

Distribution of typhoid fever prevalence rate by year in Jimma zone, Oromia region South West Ethiopia, 2015-2019 


\section{Month VS Year}

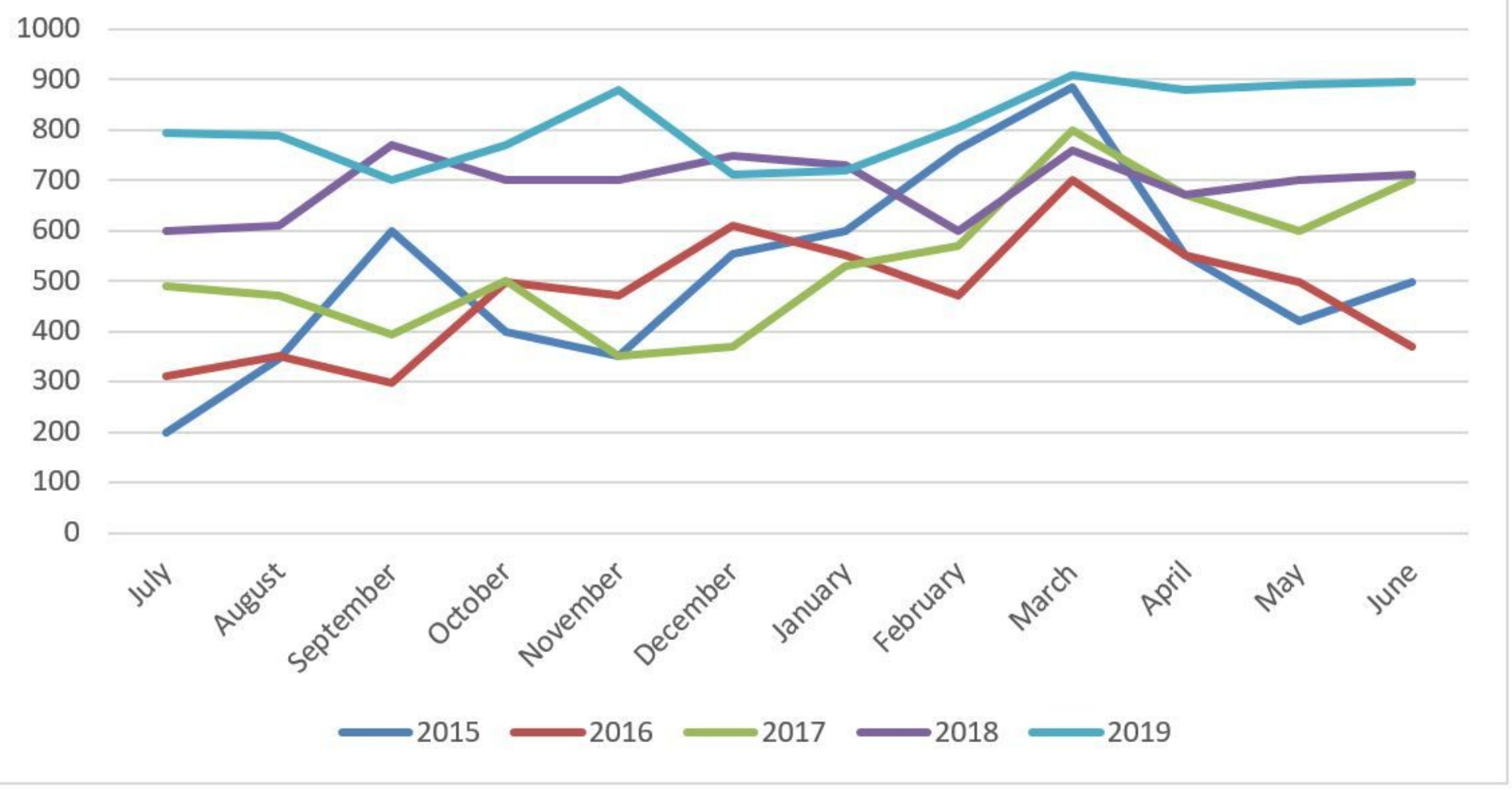

\section{Figure 3}

Trends of Typhoid Fever by month in Jimma Zone, Oromia Region, South West Ethiopia from 2015 to 201

\section{Typhoid fever case by woreda}

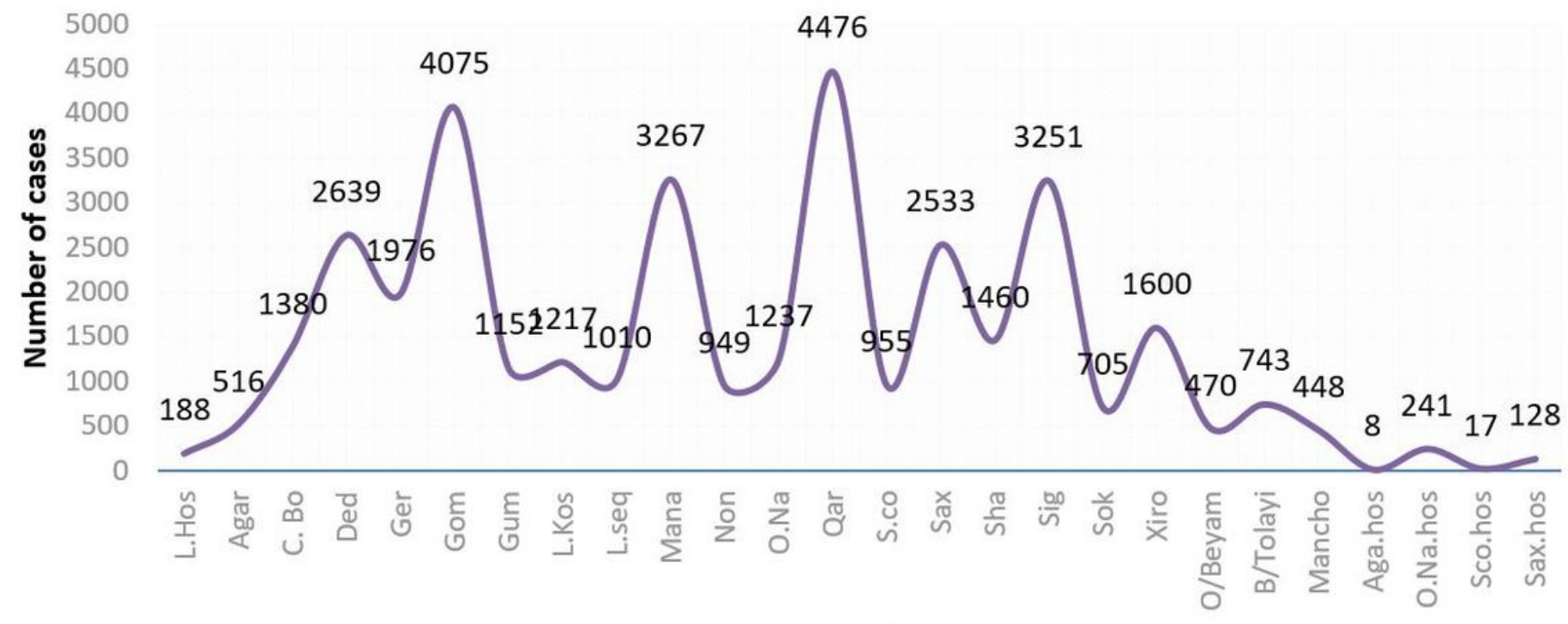

woreda 


\section{Figure 4}

Yearly trend of typhoid fever case by woreda in Jimma Zone, Oromia Region, South West Ethiopia from 2015 to 2019

\section{Morbidity by Woreda}

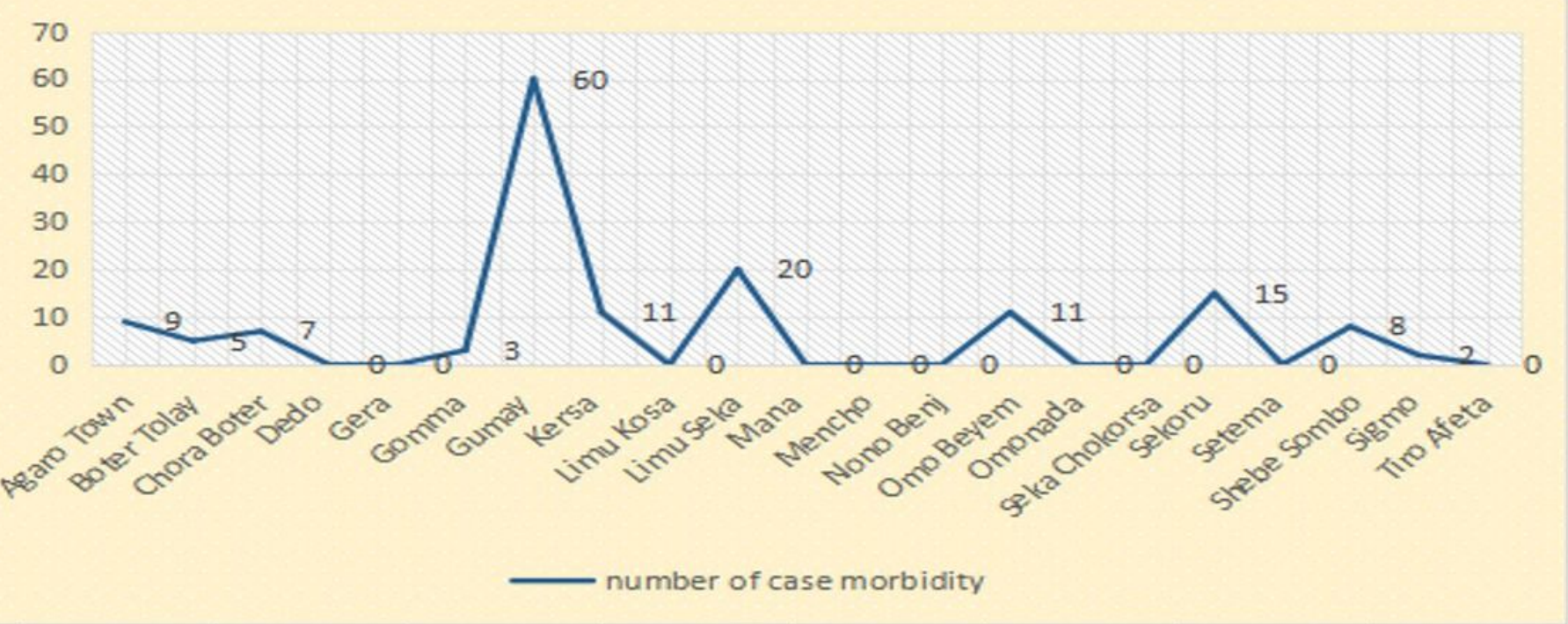

\section{Figure 5}

The distribution of Typhoid Morbidity by Woreda / place in Jimma Zone South West

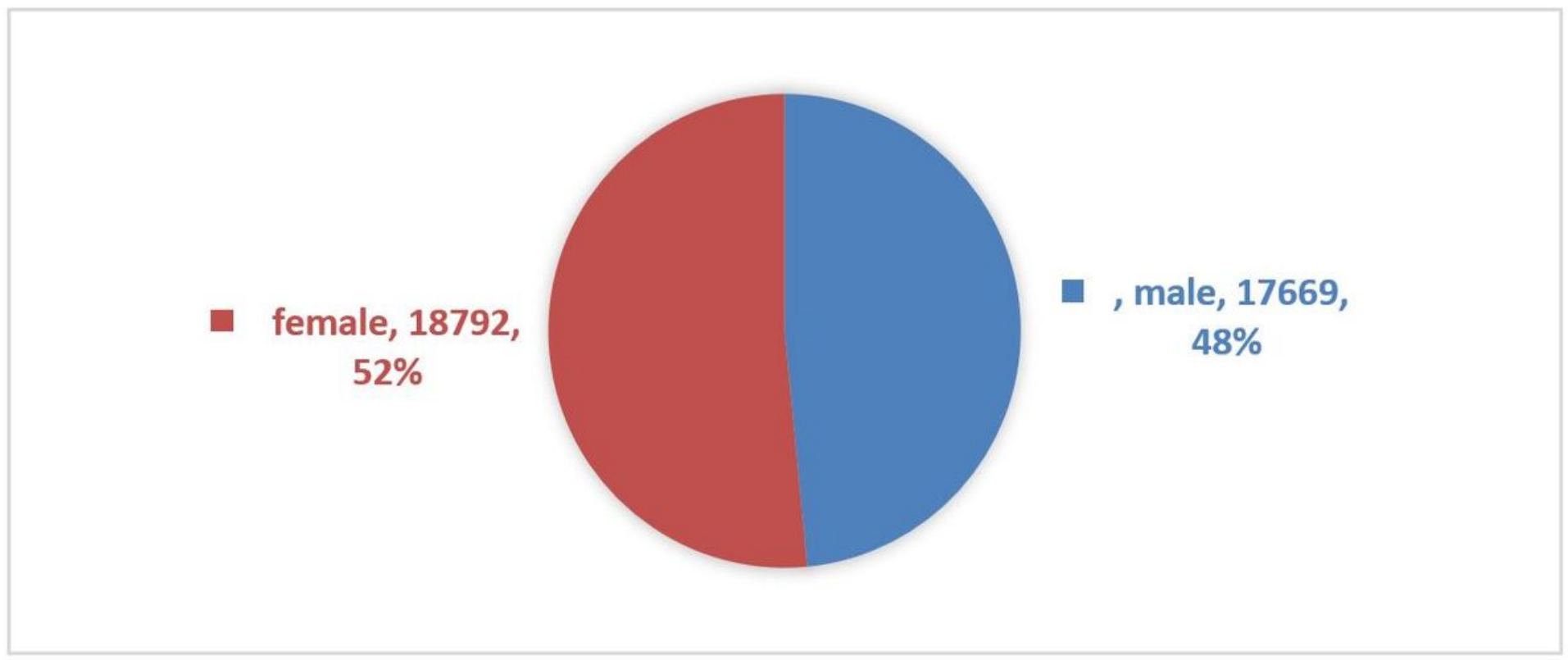

Figure 6

Typhoid fever case distribution in sex 


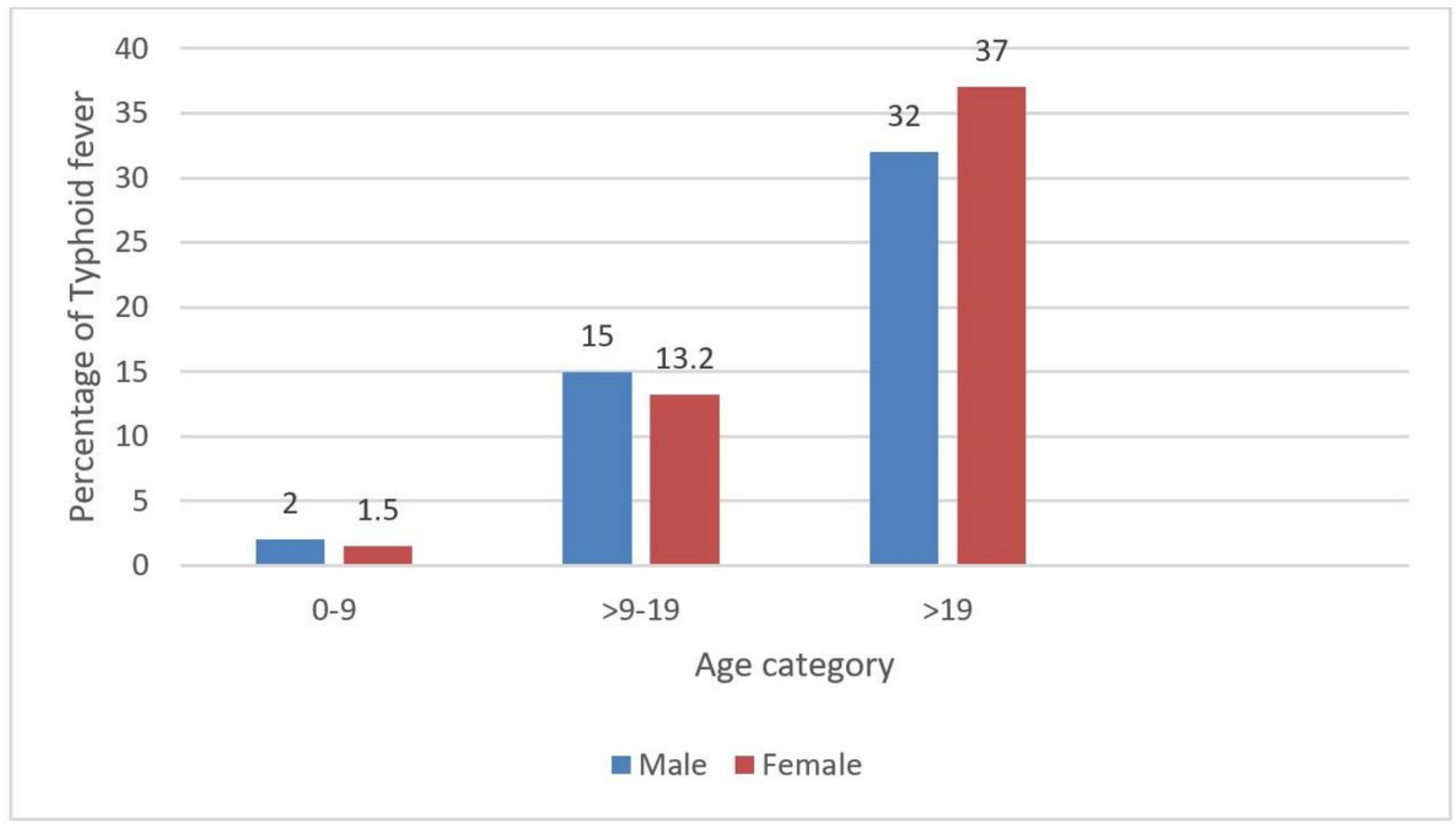

Figure 7

Distribution of typhoid fever case by age group in Jimma Zone, Oromia Region, South West Ethiopia from 2015 to 2019 